\title{
A Brief Analysis of the Effective Application of Power Electronic Devices in Power System
}

\author{
Gao Xueqian \\ North China Electric Power University (Baoding), Institute of international education, Baoding, Hebei, \\ 071003, China \\ gapxueqian@126.com
}

Keywords: Power system, power electronic device, power storage, transmission link

\begin{abstract}
The power electronic device is an integral part of the power system, is directly related to its overall performance, and determines whether the power system can achieve intelligence or not. At the present stage, the power transmission engineers regard the transmission capability of HV AC system as the main concern to ensure stable and reliable operation in power grid. The advantage of the power electronic device is to reduce the uncertainty in the operation of the power system by relying on the DC transmission technology. Based on the simple analysis and introduction of power system and power electronic devices, the application of power electronic devices in power generation, power storage and transmission links is discussed in detail so as to ensure the overall operation quality and efficiency of power system in practice.
\end{abstract}

\section{Introduction}

Power electronic devices are often preferred in the process of power system operation and development because of their technical and functional advantages, and its role can not be ignored. The related research shows that most users can use power electronic devices to deal with electricity once or more times when they use power. HVDC transmission is often applied to long-distance and large capacity transmission because of its superiority, and the converter is often used as the best choice for the rectifier valve and the receiving valve of the receiving terminal. According to the actual situation of the power system, the power engineers should choose and apply the power electronic devices reasonably so as to achieve a good power generation effect.

\section{Brief introduction of power system}

The power system is closely related to the lives of the people. It is necessary to use the system in the process of energy utilization, transportation, and distribution. At present, China's fossil energy is increasingly depleted, and there are many uncertainties in the climate environment. In order to achieve sustainable development, the power system gradually presents the characteristics of intellectualization. Different from the past, main grid, local power grid and micro grid are indispensable. During the operation of power system, more and more energy storage devices and distributed power sources are used, and the transmission process is more convenient. The transmission mode is more flexible and simple. The daily distribution and user power consumption of an electric power company are characterized by intellectualization. The situation of power failure and power failure is less and less, and the quality and reliability of power supply have been greatly improved.

Many functions of the power system are based on power electronic devices. Not only the power conversion of energy storage devices, but also the flexible interconnection of AC and DC power grids, it is necessary to rely on this device so that it can be carried out normally. Nowadays, the improvement of technology level, the upgrading of high-power electrical appliances and high-voltage power electronic devices, the modularization and unitized characteristics of converters become more 
and more prominent. The power system is more optimized by [1] with the support of power electronic devices.

\section{Development of power electronics technology}

Generally, power electronic technology mainly refers to the manufacturing devices or the use of electrical and electronic circuits. At the beginning of the development, the power electronics belongs to the semi controlled type. With the innovation of technology and technology, the power electronics gradually evolved into full control and compound type. It has developed to the present, has realized the unification of the drive, control and circuit protection. The power integrated circuit is formed from this, and its greatest advantage is that the power is small.

Both the rectifier circuit and the inverter circuit are widely used in the power electronic circuit, especially the rectifier circuit is the main application mode. The operation and development of power system provide wider application space for turn off devices. At the same time, power electronic circuits also present high frequency characteristics. New circuit topology represented by resonant inverter circuit arises at the historic moment. PWM control provides a broad opportunity for the development of power electronics technology, and other kinds of control methods provide an unlimited prospect for the technology. At present, the operation and development of power system, more inclined to choose the microprocessor based digital control technology, because of its obvious advantages in the industry in favor of [2].

\section{Application of power electronic device in power system}

\subsection{Application of power electronic device in power generation}

(1) The excitation of the generator set

In the current technical background, many large generators have eliminated exciter, and they tend to choose more functional excitation technology. Both in the power system control, or the speed of adjustment, all have obvious advantages. If this technology is applied to the operation of the unit, the operation efficiency and the overall performance of the power plant will be greatly improved. Take the hydraulic generator, by using the dynamic change form of excitation current frequency, while relying on the AC excitation technology, performance optimization of power system, ensure that the latter can quickly adjust water pressure and water flow dynamic changes, enhance two-way power system quality and efficiency.

(2) Wind power generation

In the process of wind power generation, it is bound to be inseparable from the AC. Its application principle is as follows: with the rectifier and inverter as the carrier, the original wind energy exists in the form of electric energy, and the electrical energy is consistent with the grid connection requirements in terms of voltage, phase and frequency. The topology of the converter is relatively complex. It was initially two level or three level topology, and then gradually developed into a multilevel topology. There are many multilevel topology categories, including the cascaded $\mathrm{H}$ bridge, the modular multilevel converter and so on. At present, wind power generation system has developed significantly, no matter capacity or voltage level, not only the line loss has been reduced, but also the transmission line cost is less and less. According to the regional characteristics and energy advantages, the power company should try the form of wind power to increase the development of this module. If we use the wind power generation system in the power grid, we must focus on active power control and reactive power regulation. We should not only pay attention to the change of grid connection frequency, but also consider faults and low voltage ride through performance. The use of wind power generation should be rigorous and prudent [3].

(3) Photovoltaic power station

The function of the photovoltaic power station is mainly based on the solar energy, and the centralized use of solar energy is the normal state in the process of the use of the equipment. The main 
components involved include the confluence, the boost transformer, the filter, the photovoltaic array component, the inverter group and so on. The application principle of photovoltaic power station is to give the parallel inverter as the carrier and select the best control method, and maximize its dynamic grid compensation function and reactive power compensation function. In fact, there are still many technical shackles in the practical application of large photovoltaic power plants, such as hot spot effect and poor combination state of inverter, which are still in research and trial stage, and have not been widely promoted. Therefore, when designing PV power plants, we should be forward-looking. We should take all factors into consideration and observe whether photovoltaic array, inverter combination and grid connected topology are related to the technology.

\subsection{Application of power electronic device in energy storage}

(1) cCompressed air energy storage

The innovation of technology provides a research space for compressed air energy storage, and the system has a promising future. The main application principle of compressed air energy storage is to control the air in the gas storage space starting with the electric charge of the power system. When the electric peak is used in the peak period, the pressure of the power system is relatively large, and it is easy to have various kinds of faults. However, in the background of this technology, the excess power will act on the air compressor and drive it to act. Through this way, the energy transformation can be realized, so that the excess electricity will be stored in the form of high pressure air and stored. Once the peak period of power consumption is applied, the power system is faced with a large load. The high pressure air in the storage space is released immediately, and the generator is driven again, so that it can work normally. The reason that compressed air energy storage can adjust the load of power grid is mainly because of its use of frequency conversion technology. The technology is in accordance with the energy storage requirements, and the efficiency of air compression is very high. The best way to improve the efficiency of power generation is to use the control of the excitation of the generator to further broaden the range of [4].

(2) Adjustable speed pumped storage energy

In the power system, the application of pumped storage energy needs the fall of the above reservoir as the support, so as to control the power generation system. In the course of concrete practice, in order to achieve the best effect of power generation, the consideration of the drop and speed change of the upper and lower reservoirs is necessary. The application principle of adjustable pumped storage is to select the excitation form of rotor group, choose one of them based on thyristor based cycle converter or voltage controlled and current mode based on total control, and control voltage and current. The application principle of energy storage is more complex, professional and strong, the frequency and amplitude of the rotor excitation current as the turning point, independent regulation of active and reactive power output, startup mode and operation mode switching unit, make the power system more stable, effective, both the peak and valley load, or the frequency and phase are more convenient.

(3) Battery energy storage

The use of a lithium ion battery or a sodium ion battery in the application of a power electronic device in a battery energy storage is large. The principle of its application is to balance the current of the battery module with a small power DC/DC converter as the carrier. In any battery module, the high-power DC/DC converter is integrated, and the battery module output interface is connected in series or parallel. This not only helps to improve the voltage level of the DC bus, but also can upgrade the topology of the power regulation system to make it more advantageous.

\subsection{Application of power electronic device in micro grid}

The essence of the micro grid is the small power generation system, and its structure is complex. The main application principle is to balance the local power by adjusting the power converter to establish contact with the external grid and to operate in the form of grid. When the external power grid fails, it will provide an independent operation environment for microgrid and achieve power supply for key loads, with the help of converter decomposition. Power engineers can not only 
interconnect distributed power and energy storage devices through multiple converters, but also use a single multi interface converter to achieve this function. Compared with the two, the multi interface converter has more advantages, which can connect all kinds of distributed power supply, load, energy storage device and so on. It applies the integrated control system, and has various operation modes, including three kinds of production, emergency and recovery. It not only can transfer reference values and control quantities inside the controller, but also can carry out mode transition. The speed and accuracy of transmission are very high. At the same time, the converter can handle or dispatch the DC or AC power of an interface with a great economic benefit [5].

\subsection{Application of power electronic device in transmission link}

(1) Frequency division transmission

In some power generation systems, the rotation speed of the generator is relatively low. For example, wind power, water power and so on. It often takes the frequency division transmission method as the best choice. In the process of power transmission, the frequency transmission method is often used. The transmission frequency is always low. Under this condition, the electrical distance of AC transmission lines is also shortened, and the transmission efficiency has been significantly improved. Meanwhile, the fluctuation of voltage will also be significantly reduced.

(2) Direct current transmission

DC transmission has a wide range of applications because of its technical characteristics. It is used in both urban power supply and renewable energy generation. DC power generation can be divided into two kinds of conventional direct current transmission and flexible DC transmission. The main carrier of the former is the thyristor converter, and the latter is realized by a fully controlled device converter. Flexible HVDC can not only control the active power independently, but also control the reactive power independently. The whole process needs no filtering, reactive power compensation and passive load power supply.

(3) Solid state transformer

In the actual production and living process, the demand for electric energy quality is increasing day by day. The original dynamic voltage recovery period, static synchronous compensator and other power quality compensation devices have been unable to meet the control requirements of power quality due to their single function and low efficiency. The technical advantage of solid state transformer lies in its high cost performance, its own reactive power compensation function, and it can adjust the amplitude or phase characteristics of voltage or current with the help of two technologies of power electronics conversion and electromagnetic coupling power transformation. Whether the voltage rises, falls, harmonics, flicker, or the unbalanced three-phase output voltage, it is able to respond flexibly. Solid state transformer not only can control the power flow, and according to the actual need to adjust the power quality, strong stability, ensure that the transmission process flexibility, so that the power transmission process more controllable, and can guarantee the performance of power system and the power transmission efficiency and quality. At the same time, it can also cut off the fault current in a very short time to realize the balance of the function of the circuit breaker [6].

\section{Conclusions}

To sum up, the application of power electronic devices in power system is very advantageous, which makes the power system safer and more reliable during operation, and its control process is more automated. The application of power electronic equipment in power system should not only stay in the range and the way, should also be more from the aspects of operation, reliability, fault simulation and circuit considerations, technical research and technological innovation and improve the overall quality of the operation of power system in our country, to provide a good environment for people with electricity. 


\section{References}

[1] Gao Xuelin. power electronic equipment in power system application of [J]. electronic world, 2014 (18): 71-71.

[2] Yu Xiang. Research on the application of power electronic devices in power system [J]. electronic technology and software engineering, 2015, (10): 119-119.

[3] Zhu Chunlei. Research on the main application of power and electronic devices in power system of power plant [J]. automation and instrumentation, 2017, (7): 222-223.

[4] Zhao Xinchuan, Meng Xuebin, et al. Practical application of power electronic devices in power systems analysis [J]. electronic production, 2017, (7): 90-91.

[5] Wu Fang. High-power power electronic devices in power system test study [J]. electronic production, 2014, (20): 13-14.

[6] Jiang Yingwei, Hou Kai, and other applications of. Ether CAT bus technology in modern power electronic devices [J]. China electric power, 2016, 49 (9): 66-71. 